\title{
Atrial Enlargement by ECG Finding
}

National Cancer Institute

\section{Source}

National Cancer Institute. Atrial Enlargement by ECG Finding. NCI Thesaurus. Code C71039.

An electrocardiographic finding which comprises left, right or bilateral atrial enlargement. This is may be characterized by prolong ed $\mathrm{P}$ wave duration, increased $\mathrm{P}$ wave amplitude, or multi-component P waves. (CDISC) 